\title{
Repérer et prendre en charge les personnes âgées en pratique clinique : nous ne devons plus attendre
}

\section{Identify and support the elderly in clinical practice: we can not wait any longer}

\author{
B. Vellas $\cdot$ S. Sourdet $\cdot$ P. Cestac $\cdot$ S. Oustric $\cdot$ J.-E. Morley \\ C) Springer-Verlag France 2012
}

Dans les années 1970, l'hospitalisation dans les départements des urgences de nombreux sujets âgés polypathologiques, avec un déclin cognitif et des incapacités sévères, a rendu nécessaire l'émergence d'une nouvelle spécialité dans de nombreux pays : la gériatrie. En réalité, à cette époque, personne ne souhaitait ou n'était capable de prendre en charge des sujets âgés très dépendants, nécessitant des soins au long cours. Pour cette raison, de nombreux départements de médecine gériatrique, essentiellement des services de soins subaigus, et de long séjour, ont été créés. Depuis, la médecine gériatrique s'est développée dans de nombreux pays et existe de nos jours sous la forme d'unités de soins aigus, d'hôpitaux de jour, d'équipes mobiles et de centres mémoire à travers le monde entier. Cependant, dans la plupart des centres, les médecins gériatres accueillent des patients avec des incapacités déjà sévères à un stade souvent irréversible. Près de $95 \%$ des forces de la médecine gériatrique s'orientent donc sur des sujets âgés qui sont déjà dépendants. Il est évidemment indispensable de continuer à prendre en charge ces patients, néanmoins, un nouvel axe de la médecine gériatrique doit se développer visant à prendre en charge les patients qui ne sont pas encore dépendants mais à risque de le devenir : les sujets fragiles et préfragiles. Il s'agit d'une absolue nécessité si nous

B. Vellas $(\bowtie) \cdot$ S. Sourdet

Gérontopôle, CHU Toulouse

e-mail : vellas.bruno@gmail.com

B. Vellas $\cdot$ P. Cestac

INSERM UMR 1027

P. Cestac

Pôle pharmacie, CHU Toulouse

S. Oustric

Département universitaire de médecine générale

J.-E. Morley

Saint Louis University, USA

Co-édition avec L'Année gérontologique (2012) voulons prévenir et anticiper l'accroissement rapide de la dépendance dans notre population vieillissante et promouvoir des soins plus efficients.

Les sujets âgés fragiles et préfragiles sont ceux qui, selon les critères de Fried, ont une perte de poids involontaire, une grande sédentarité, une fatigue générale, une faible force musculaire et une vitesse de marche lente [1-7]. Si ces personnes ont au moins un des critères de Fried, les sujets sont préfragiles; s'ils ont trois critères ou plus, ils sont fragiles. Les sujets âgés fragiles sont plus à risque de devenir dépendants, pourtant aujourd'hui, ils ne sont pas réellement pris en charge par notre système de soins. Il s'agit donc, en collaboration avec les médecins de ville, de relever ce challenge. Dans ce but, nous devons proposer une intervention ciblée, forte, et soutenue.

\section{Cibler les sujets âges fragiles et préfragiles}

Pour cela, nous avons besoin d'un outil valide et simple à utiliser pour les médecins généralistes et les autres professionnels de santé. Le questionnaire FRAIL (cinq questions seulement), développé par « The IANA Task Force » $[5,6]$ et récemment validé par Morley et al. est un bon exemple [4]. Un autre exemple est l'outil utilisé au sein de la plateforme expérimentale d'évaluation des fragilités et de prévention de la dépendance du Gérontopôle (voir article J. Subra et collaborateurs). Il est ici utile d'y associer l'évaluation subjective du médecin afin qu'il reste impliqué dans le processus d'intervention. Environ $95 \%$ des sujets adressés à la plateforme du Gérontopôle à l'aide de cet outil de screening sont fragiles ou préfragiles

\section{Une intervention forte}

Pour avoir un réel impact, l'intervention doit être forte.Une évaluation gériatrique complète des patients préfragiles et fragiles doit permettre de diagnostiquer les maladies liées à l'âge 
à un stage prodromal, où il est encore possible d'agir de façon efficace. La détection des stades précoces de la dégénérescence maculaire, du glaucome, des troubles auditifs, du déclin cognitif léger, de la sarcopénie [8,9] ou de la perte de mobilité notamment, doit être une priorité et s'appuyer sur des outils spécifiques. Une évaluation globale : médicale, psychologique, sociale, économique, ainsi que de l'accumulation des déficits [10] doit être réalisée. De plus, cette évaluation doit être aussi l'opportunité d'impliquer les patients dans la recherche clinique, de leur proposer des essais thérapeutiques avec de nouvelles molécules comme il en existe par exemple dans la maladie d'Alzheimer prodromale ou la sarcopénie.

\section{Une intervention soutenue et prolongée}

Dans cette population qui va continuer à vieillir, il est nécessaire de maintenir une intervention soutenue sur le long terme. À la détection et au traitement des maladies liées à l'âge, doivent s'associer l'exercice physique, cognitif, les interventions nutritionnelles et sociales. Une étude récente a montré que même chez les sujets âges fragiles après une fracture de la hanche, un programme d'exercice musculaire de haute intensité associé à une intervention multidisciplinaire pouvait améliorer la mortalité, l'entrée en institution et la dépendance à un an [11]. Une standardisation de ces interventions multidomaines est un domaine important de la recherche dans le futur. Nous avons besoin de trouver un compromis entre des interventions très fortes qui seront acceptées que par un petit nombre de sujets âgés fragiles et des interventions trop légères qui ne pourront pas avoir d'impact réel. Le développement des activités de la vie quotidienne doit être encouragé.

- L'IAGG (International Association of Gerontology and Geriatrics), http://www.iagg.info/ and the G.A.R.N. (IAGG Global Aging Research Network) http://garnnetwork.org/index.php a déjà pris et prendra davantage d'initiatives dans ce domaine [12]. Les membres de l'IAGG ont pointé la nécessité de concentrer sur le " agingin-place » pour prévenir des placements en maison de retraite trop précoces. L'évaluation de la fragilité et de la préfragilité dans les soins gériatriques usuels est donc devenue un enjeu mondial. Un article de consensus suite au workshop d'Athènes réalisé par la SFCG (Pr G. Berrut) et l' IAGG (Pr A. Benetos) est en cours de publication.

Si nous sommes capables de reconnaître et traiter la fragilité dans notre pratique clinique courante, s'ouvre alors une nouvelle ère de la médecine gériatrique. Dès lors, nous serons capables de développer une recherche clinique de haut niveau impliquant une approche autour des biomarqueurs, de l'imagerie, et de nouvelles thérapeutiques plus ciblées. Des interventions multidomaines ou multimodales seront à envisager. De plus, des actions devront être mises en œuvre pour prévenir la dépendance iatrogène si ces sujets âgés fragiles sont amenés à être hospitalisés [13]. Cette ouverture de la médecine gériatrique vers l'évaluation de la préfragilité et la fragilité sera coût-efficiente et peut offrir un nouveau rebond pour la discipline, comme cela est suggéré dans les récentes initiatives européennes $[14,15]$.

\section{Références}

1. Fried LP, Tangen CM, Walston J, Newman et al (2001) Cardiovascular Health Study Collaborative Research Group. Frailty in older adults: Evidence for a phenotype. J Gerontol A Biol Sci Med Sci 56:M146-M156

2. Abellan van Kan G, Rolland Y, Houles M, et al (2010) The assessment of frailty in older adults. Clin Geriatr Med 26:275-86

3. Abellan van Kan G, Rolland Y, Andrieu S, et al (2009) International Academy on Nutrition and Aging (IANA) Task Force. Gait speed at usual pace as a predictor of adverse outcomes in community-dwelling older people. J Nutr Health Aging 13:881-9

4. Morley JE, Malmstrom TK, Miller DK (2012) A simple frailty questionnaire (FRAIL) predicts outcomes in middle aged African Americans. J Nutr Health Aging (in press)

5. Abellan van Kan G, Rolland YM, Morley JE, Vellas B (2008) Frailty: Toward a clinical definition. J Am Med Dir Assoc 9:71-2

6. Abellan van Kan G, Rolland Y, Bergman H, et al (2008) The I.A.N.A. Task Force on frailty assessment of older people in clinical practice. J Nutr Health Aging 12:29-37

7. Nourhashemi F, Andrieu S, Gillette-Guyonnet S, et al (2001) Instrumental activities of daily living as a potential marker of frailty: A study of 7364 community-dwelling elderly women (the EPIDOS study). J Gerontol A Biol Sci Med Sci 56:M448-M453

8. Chumlea WC, Cesari M, Evans WJ, et al (2011) International Working Group on Sarcopenia Task Force Members. Sarcopenia: Designing phase IIB trials. J Nutr Health Aging 15:450-5

9. Fielding RA, Vellas B, Evans WJ, et al (2011) International Working Group on Sarcopenia. Sarcopenia: An undiagnosed condition in older adults. Current consensus definition: Prevalence, Etiology, and consequences. J Am Med Dir Assoc 12:249-56

10. Rockwood K, Mitnitski A (2012) How might deficit accumulation give rise to frailty? J Frailty Aging 1:8-12

11. Singh NA, Quine S, Clemson LM, et al (2012) Effects of highintensity progressive resistance training and targeted multidisciplinary treatment of frailty on mortality and nursing home admissions after hip fracture: A randomized controlled trial. J Am Med Dir Assoc 13:24-30.

12. Tolson D, Rolland Y, Andrieu S, (2011) The International Association of Gerontology and Geriatrics/World Health Organization/ Society Française de Gérontologie et de Gériatrie Task Force. International Association of Gerontology and Geriatrics: A global agenda for clinical research and quality of care in nursing homes. J Am Med Dir Assoc 12:184-9

13. Lafont C, Gérard S, Voisin T, Pahor M, Vellas B (2011) Members of I.A.G.G./A.M.P.A. Task Force. Reducing "iatrogenic disability" in the hospitalized frail elderly. J Nutr Health Aging 15:645-60

14. Rodriguez-Mañas L, Féart C, Mann G, on behalf of the FOD-CC group et al (2012) (Appendix 1). Search for an Operational definition of frailty: A Delphi method based consensus statement. The Frailty Operative Definition-Consensus conference project. J Gerontol A Biol Sci Med Sci 67

15. Cesari M (2012) Frailty and Aging J Frailty Aging 1:3-6 